\title{
Application of some new contractions for existence and uniqueness of differential equations involving Caputo-Fabrizio derivative
}

\author{
Hojjat Afshari ${ }^{1}$, Hossein Hosseinpour ${ }^{2}$ and H.R. Marasi2 ${ }^{*}$
}

"Correspondence:

marasi@tabrizu.ac.ir

${ }^{2}$ Department of Applied

Mathematics, Faculty of

Mathematical Sciences, University

of Tabriz, Tabriz, Iran

Full list of author information is

available at the end of the article

\begin{abstract}
In this paper we study fractional initial value problems with Caputo-Fabrizio derivative which involves nonsingular kernel. First we apply $\alpha-\ell$-contraction and $\alpha$-type $F$-contraction mappings to study the existence and uniqueness of solutions for such problems. Finally, we use some contraction mappings in complete $\mathfrak{F}$-metric spaces for this purpose.
\end{abstract}

Keywords: Caputo-Fabrizio fractional derivative; $\mathfrak{F}$-metric space; $\alpha$ - $\ell$-contraction mapping; Fixed point

\section{Introduction}

Fractional calculus is a part of mathematical analysis that studies the performance of derivative and integral operations on non-integer orders. In the past years, early work in fractional calculus was limited to mathematics. But, in the last few years, extensive studies on the applications of fractional operators in the other disciplines have been conducted. Recently, this field had found many applications in various directions such as applied mathematics, electrochemistry, tracer in fluid flows, fractional-order multi-poles in electromagnetism, finance, signal processing, bio-engineering, viscoelasticity, fluid mechanics, and fluid dynamics [10, 24]. These wide applications have led researchers to provide different definitions of fractional derivatives. The main difference between these definitions is related to possessing different kernels. Two famous fractional derivatives, namely the Riemann-Liouville and the Caputo derivatives, have received a lot of attention and so differential and integral equations containing these derivatives by several methods containing numerical and analytical methods $([22,29,30]$, but these definitions included a singular kernel. Thus, recently Caputo and Fabrizio provided a definition with a nonsingular kernel which the properties of this new definition can be found in [25]. Various methods have been used by researchers to solve differential equations including Caputo-Fabrizio fractional derivative and multi-singular point-wise defined equations (see $[16,18,32]$ and the references therein). One of the efficient methods in investigating the existence and

(c) The Author(s) 2021. This article is licensed under a Creative Commons Attribution 4.0 International License, which permits use, sharing, adaptation, distribution and reproduction in any medium or format, as long as you give appropriate credit to the original author(s) and the source, provide a link to the Creative Commons licence, and indicate if changes were made. The images or other third party material in this article are included in the article's Creative Commons licence, unless indicated otherwise in a credit line to the material. If material is not included in the article's Creative Commons licence and your intended use is not permitted by statutory regulation or exceeds the permitted use, you will need to obtain permission directly from the copyright holder. To view a copy of this licence, visit http://creativecommons.org/licenses/by/4.0/. 
uniqueness of the solutions of differential equations is using of fixed point theory and for this reason there is a long history of presenting various fixed point theorems (see [1$7,9,11,13,17,19,22,26-28,34])$.

In 2012, Samet et al. [33] proposed the concept of $\alpha-\psi$ contractive mappings and investigated the existence of fixed points for such mappings, then some researchers improved them to large classes of the contractive type mappings (see [8, 12, 14, 15, 20, 22, 23]).

Wardowski et al. [35] proposed and investigated the $F$-contraction, then Abbas et al. $[17,21]$ further generalized the concept of $F$-contraction and proved some fixed point results.

Wasfi Shatanawi and Erdal Karapınar in [34] introduced $F_{S}$-contractions in the sense of Wardowski and Seghal and $F_{J}$-contractions in the sense of Wardowski and Jachymski. Then they ensured some existence and uniqueness fixed point results. Throughout the article $\mathfrak{J}$ denote $[0,1]$.

In this work, we consider the following differential equation with Caputo-Fabrizio derivatives via fixed point theorems:

$$
\begin{aligned}
& \left(\begin{array}{l}
C F \\
0
\end{array} D^{\varsigma} \hbar\right)(p)=f(p, \hbar(p)), \quad p \in \mathfrak{J}, 0<\varsigma<1, \\
& \hbar(0)=\hbar_{0},
\end{aligned}
$$

where $D^{\varsigma}$ is the Caputo-Fabrizio derivative of order $\varsigma$ and $f$ is continuous with $f(0, \hbar(0))=0$.

In what we will have below it is supposed that $(M, d)$ be a complete $b$-metric space and $p_{1}$ is its constant, also the elements of $\Omega$ are increasing and continuous functions $\ell:[0, \infty) \rightarrow[0, \infty)$ satisfying $\ell(q x) \leq q \ell(x) \leq q x, q>1$; moreover, $\Lambda$ denotes the family of nondecreasing functions such that, for $p_{1} \geq 1$, we have $\varrho:[0, \infty) \rightarrow\left[0, \frac{1}{p_{1}^{2}}\right)$.

Definition 1.1 ([5]) Let $\psi: M \rightarrow M$ and suppose that there exists $\alpha: M \times M \rightarrow[0, \infty)$ with

$$
\alpha(x, y) \ell\left(p_{1}^{3} d(\psi x, \psi y)\right) \leq \varrho(\ell(d(x, y))) \ell(d(x, y)),
$$

for $x, y \in M, \varrho \in \Lambda$ and $\ell \in \Omega$. Then $\psi$ is called a generalized $\alpha$ - $\ell$-Geraghty contraction mapping.

Definition $1.2([33])$ Let for $\psi: M \rightarrow M$ where $M \neq \emptyset$ and $\alpha: M \times M \rightarrow[0, \infty)$ we have

$$
\alpha(x, y) \geq 1 \quad \Longrightarrow \quad \alpha(\psi x, \psi y) \geq 1, \quad \forall x, y \in M
$$

Then $\psi$ is called an $\alpha$-admissible mapping.

Now, we have the following fixed point theorem.

Theorem 1.3 ([5]) Let $\psi: M \rightarrow M$ be a generalized $\alpha$ - $\ell$-Geraghty and

(n1) $\psi$ is $\alpha$-admissible;

(n2) there exist $\jmath_{0} \in M$ with $\alpha\left(J_{0}, \psi_{J_{0}}\right) \geq 1$; 
(n3) $\left\{J_{n}\right\} \subseteq M, \lim _{n \rightarrow \infty} J_{n}=u$ where $u \in M$ and $\alpha\left(J_{n}, J_{n+1}\right) \geq 1$

implies $\alpha\left(J_{n}, J\right) \geq 1$.

Then there exists a fixed point for the mapping $\psi$.

Definition 1.4 Let $0<\varsigma<1, j \in C^{1}[0, b), b>0$. The Caputo-Fabrizio derivative for $J$ of order $\varsigma$ is defined by

$$
{ }^{C F} D^{\varsigma} J(p)=\frac{M(\varsigma)(2-\varsigma)}{2(1-\varsigma)} \int_{0}^{p} \exp \left(-\frac{\varsigma}{1-\varsigma}(p-x)\right) J^{\prime}(x) d x, \quad s \geq 0
$$

where $M(\varsigma)$ is a normalization constant depending on $\varsigma$ with $M(0)=M(1)=1$. Note that $\left({ }^{C F} D^{\varsigma}\right)(J)=0$ if and only if $J$ is a constant.

Definition 1.5 Let $0<\varsigma<1$. The Caputo-Fabrizio integral for a function $J$ of order $\varsigma$ is defined by

$$
{ }^{C F} I^{\varsigma}(J)(p)=\frac{2(1-\varsigma)}{M(\varsigma)(2-\varsigma)} J(p)+\frac{2 \varsigma}{(2-\varsigma) M(\varsigma)} \int_{0}^{p} J(x) d x, \quad p \geq 0 .
$$

Take as $d: M \times M \rightarrow[0, \infty)$ given by

$$
d(\hbar, \wp)=\left\|(\hbar-\wp)^{2}\right\|_{\infty}=\sup _{p \in \mathfrak{J}}(\hbar(p)-\wp(p))^{2},
$$

where $p_{1}=2$ is the constant of $(M, d)$ and $M=\mathcal{C}(\mathfrak{J}, R)$.

In this paper we consider

$$
\begin{aligned}
& \left(\begin{array}{l}
C F \\
0
\end{array} D^{\varsigma} \hbar\right)(p)=f(p, \hbar(p)), \quad p \in \mathfrak{J}, 0<\varsigma<1, \\
& \hbar(0)=\hbar_{0},
\end{aligned}
$$

where $D^{\varsigma}$ is the Caputo-Fabrizio derivative of order $\varsigma$, also it is supposed that $f: \mathfrak{J} \times M \rightarrow$ $M$ satisfies in $f(0, \hbar(0))=0$ and is continuous.

It is easy to prove the following lemma.

Lemma 1.6 If $0<\varsigma<1$, then

$$
\left({ }^{C F} I_{b}^{\zeta C F} D^{\varsigma} \hbar\right)(p)=\hbar(p)-\hbar(b) .
$$

\section{Main results}

In this section, for existence and uniqueness of a solution for the problem be defined in (6) firs we apply an $\alpha$ - $\ell$-contraction, then we continue by using an $\alpha$-type $F$-contraction and another contraction in complete $\mathfrak{F}$-metric space to examine the existence and uniqueness of solutions of the mentioned problem.

\section{Theorem 2.1 Suppose}

(n1) there exist $\mathrm{J}: R^{2} \rightarrow R$ such that

$$
|f(p, \hbar(p))-f(p, \wp(p))| \leq \frac{(2-\varsigma) M(\varsigma)}{4 \sqrt{2}} \sqrt{\varrho\left(\ell\left(|\hbar(p)-x(p)|^{2}\right)\right) \ell\left(|\hbar(p)-\wp(p)|^{2}\right)}
$$


for $p \in \mathfrak{J}, \ell \in \Omega$ and $\hbar, \wp \in \mathcal{C}(\mathfrak{J}, R)$ with $J(\hbar, \wp) \geq 0$;

(n2) there exist $\hbar_{1} \in \mathcal{C}(\mathfrak{J})$ with $J\left(\hbar_{1}(p), \top \hbar_{1}(p)\right) \geq 0, p \in \mathfrak{J}$, where $\top: \mathcal{C}(\mathfrak{J}) \rightarrow \mathcal{C}(\mathfrak{J})$ is defined by

$$
(\top \hbar)(p)=\hbar_{0}+{ }_{0}^{C F} I^{\varsigma} f(p, \hbar(p))
$$

(n3) for $p \in \mathfrak{J}$ and $\hbar, \wp \in \mathcal{C}(\mathfrak{J}), J(\hbar(p), \wp(p)) \geq 0$ implies $J(T \hbar(p), T \wp(p)) \geq 0$;

(n4) $\left\{\hbar_{n}\right\} \subseteq \mathcal{C}(\mathfrak{J}), \hbar_{n} \rightarrow \hbar$ where $\hbar \in \mathcal{C}(\mathfrak{J})$ and $J\left(\hbar_{n}, \hbar_{n+1}\right) \geq 0$ implies $J\left(\hbar_{n}, \hbar\right) \geq 0$, for $n \in \mathrm{N}$.

Then there exist at least one solution for the problem (6).

Proof Applying the Caputo-Fabrizio integral and using Proposition 1.6, from (6) we have

$$
\hbar(p)=\hbar_{0}+{ }_{0}^{C F} I^{\varsigma} f(p, \hbar(p))=\top \hbar(p) .
$$

We prove that $T$ has a fixed point. Thus,

$$
\begin{aligned}
& |T \hbar(p)-T \wp(p)|^{2} \\
& =\left|{ }_{0}^{C F} I^{k}[f(p, \hbar(p))-f(p, \wp(p))]\right|^{2} \\
& \leq\left\{\mid \frac{2(1-\varsigma)}{(2-\varsigma) M(\varsigma)}[f(p, \hbar(p))-f(p, \wp(p))]\right. \\
& \left.+\frac{2 \varsigma}{(2-\varsigma) M(\varsigma)} \int_{0}^{p}[f(p, \hbar(p))-f(p, \wp(p))] d p \mid\right\}^{2} \\
& \leq\left\{\frac{2(1-\varsigma)}{(2-\varsigma) M(\varsigma)}|f(p, \hbar(p))-f(p, \wp(p))|\right. \\
& \left.+\frac{2 \varsigma}{(2-\varsigma) M(\varsigma)} \int_{0}^{p}|f(p, \hbar(p))-f(p, \wp(p))| d p\right\}^{2} \\
& \leq\left\{\frac{(2-\varsigma) M(\varsigma)}{4 \sqrt{2}} \cdot \frac{2}{(2-\varsigma) M(\varsigma)}\right\}^{2}\left\{(1-\varsigma) \sqrt{\varrho\left(\ell\left(|\hbar(p)-\wp(p)|^{2}\right)\right) \ell\left(|\hbar(p)-\wp(p)|^{2}\right)}\right. \\
& \left.+\varsigma \int_{0}^{p} \sqrt{\varrho\left(\ell\left(|\hbar(p)-\wp(p)|^{2}\right)\right) \ell\left(|\hbar(p)-\theta(p)|^{2}\right)} d p\right\}^{2} \\
& =\left\{\left(\frac{(1-\varsigma)}{2 \sqrt{2}}+\frac{\varsigma}{2 \sqrt{2}}\right)\left(\sqrt{\varrho\left(\ell\left(\sup _{p \in \mathfrak{J}}|\hbar(p)-\wp(p)|^{2}\right)\right) \ell\left(\sup _{p \in \mathfrak{J}}|\hbar(p)-\wp(p)|^{2}\right)}\right)\right\}^{2} \\
& \leq\left\{\frac{1}{2 \sqrt{2}} \sqrt{\varrho\left(\ell\left(\sup _{p \in \mathfrak{J}}|\hbar(p)-\wp(p)|^{2}\right)\right) \ell\left(\sup _{p \in \mathfrak{J}}|\hbar(p)-\wp(p)|^{2}\right)}\right\}^{2} \\
& =\left\{\frac{1}{2 \sqrt{2}} \sqrt{\varrho(\ell(d(\hbar, \wp))) \ell(d(\hbar, \wp))}\right\}^{2} \\
& =\frac{1}{8} \varrho(\ell(d(\hbar, \wp))) \ell(d(\hbar, \wp)) \text {. }
\end{aligned}
$$

Hence for $\hbar, \wp \in \mathcal{C}(\mathfrak{J}), p \in \mathfrak{J}$ with $J(\hbar(p), \wp(p)) \geq 0$, we have

$$
8\left\|(T \hbar-T \wp)^{2}\right\|_{\infty} \leq \varrho(\ell(d(\hbar, \wp))) \ell(d(\hbar, \wp)) .
$$


Put $\alpha: \mathcal{C}(\mathfrak{J}) \times \mathcal{C}(\mathfrak{J}) \rightarrow[0, \infty)$ by

$$
\alpha(\hbar, \wp)= \begin{cases}1 & \jmath(\hbar(p), \wp(p)) \geq 0, p \in \mathfrak{J} \\ 0 & \text { else }\end{cases}
$$

and

$$
\begin{aligned}
\alpha(\hbar, \wp) \ell(8 d(\top \hbar, \top \wp)) & \leq 8 d(\top \hbar, \top \wp) \\
& \leq \varrho(\ell(d(\hbar, \wp))) \ell(d(\hbar, \wp)) .
\end{aligned}
$$

So, $T$ is an $\alpha$ - $\ell$-contraction. To show that $\top$ is $\alpha$-admissible, we have from (n3)

$$
\begin{aligned}
\alpha(\hbar, \wp) \geq 1 \quad & \Rightarrow \quad J(\hbar(p), \wp(p)) \geq 0 \quad \Rightarrow \quad J(\top(\hbar), \top(\wp)) \geq 0 \\
& \Rightarrow \alpha(\top(\hbar), \top(\wp)) \geq 1,
\end{aligned}
$$

for $\hbar, \wp \in \mathcal{C}(\mathfrak{J})$. By $(\mathrm{n} 2)$, we have $\hbar_{0} \in \mathcal{C}(\mathfrak{J})$ such that $\alpha\left(\hbar_{0}, \top \hbar_{0}\right) \geq 1$. From $(\mathrm{n} 4)$ and Theorem 1.3, there exists $\hbar^{*} \in \mathcal{C}(\mathfrak{J})$ such that $\hbar^{*}=\top \hbar^{*}$.

Now to define an $\alpha$-type $F$-contraction mapping, let $\mathfrak{F}$ be the family of strictly increasing functions $F: \mathfrak{R}_{+} \rightarrow R$ such that there exists $k \in(0,1)$ for which $\lim _{\alpha \rightarrow 0^{+}} \alpha^{k} F(\alpha)=0$ and also $\lim _{n \rightarrow \infty} F\left(\alpha_{n}\right)=-\infty$ if and only if $\lim _{n \rightarrow \infty} \alpha_{n}=0$ for each sequence $\left\{\alpha_{n}\right\}_{n \in \mathrm{N}}$ of positive numbers.

Definition 2.2 Suppose that there exist $y>0, F \in \mathfrak{F}$ and $\alpha: M \times M \rightarrow\{-\infty\} \cup(0, \infty)$ such that for $\hbar, \wp \in M$ we have $d(\psi \hbar, \psi \wp)>0$ and

$$
y+\alpha(\hbar, \wp) F(d(\psi \hbar, \psi \wp)) \leq F(d(\hbar, \wp)) .
$$

Then $\psi: M \rightarrow M$ is called an $\alpha$-type $F$-contraction on $M$ where $(M, d)$ is a metric space.

We present the following theorem.

Theorem 2.3 ([17]) Let $(M, d)$ be a metric space and $\psi: M \rightarrow M$ be an $\alpha$-type Fcontraction such that:

(n1) there exist $\hbar_{0} \in M$ with $\alpha\left(\hbar_{0}, \psi \hbar_{0}\right) \geq 1$,

(n2) $\psi$ is $\alpha$-admissible,

(n3) if $\left\{\hbar_{n}\right\} \subseteq M$ with $\alpha\left(\hbar_{n}, \hbar_{n+1}\right) \geq 1$ and $\hbar_{n} \rightarrow \hbar$, then $\alpha\left(\hbar_{n}, \hbar\right) \geq 1, n \in \mathrm{N}$,

(n4) $F$ is continuous.

Then $\psi$ has a fixed point $\hbar^{*} \in M$ and for $\hbar_{0} \in M$ the sequence $\left\{\psi^{n} \hbar_{0}\right\}_{n \in \mathrm{N}}$ is convergent to $\hbar^{*}$.

If we consider the metric

$$
d(\hbar, \wp)=\sup _{t \in \mathfrak{J}}\{|\hbar(t)-\wp(t)|\},
$$

then we can prove the following theorem in $X=C(\mathfrak{J}, R)$. 
Theorem 2.4 There exist $\mathrm{J}: R^{2} \rightarrow R$ such that

(H1) $|f(p, \hbar(p))-f(p, \wp(p))| \leq \frac{(2-\varsigma) M(\varsigma)}{2} e^{-y}|\hbar(p)-\wp(p)|$ for $p \in \mathfrak{J}$ and $\hbar, \wp \in R$;

(H2) $\exists \hbar_{1} \in \mathcal{C}(\mathfrak{J})$ such that $J\left(\hbar_{1}(p), \top \hbar_{1}(p)\right) \geq 0$ for $p \in \mathfrak{J}$, where $\top: \mathcal{C}(\mathfrak{J}) \rightarrow \mathcal{C}(\mathfrak{J})$ defined by the following

$$
\top \hbar(p)=\hbar_{0}+{ }_{0}^{C F} I^{S} f(p, \hbar(p))
$$

(H3) for $p \in \mathfrak{J}$ and $\hbar, \wp \in \mathcal{C}(\mathfrak{J}), J(\hbar(p), \wp(p)) \geq 0$ implies $J(\top \hbar(p), \top \wp(p)) \geq 0$ :

(H4) $\left\{\hbar_{n}\right\} \subseteq \mathcal{C}(\mathfrak{J}), \lim _{n \rightarrow \infty} \hbar_{n}=\hbar$ where $\hbar \in \mathcal{C}(\mathfrak{J})$ and $J\left(\hbar_{n}, \hbar_{n+1}\right) \geq 0$ implies $J\left(\hbar_{n}, \hbar\right) \geq 0$, for $n \in \mathrm{N}$.

Then there exists at least one fixed point for $\top$ which is the solution of problem (6).

Proof We show that $T$ has a fixed point. Thus,

$$
\begin{aligned}
& |\mathrm{T} \hbar(p)-\mathrm{\top} \wp(p)||\top \hbar(p)-\top \wp(p)+1| \\
& =\left|{ }^{C F} I[f(p, \hbar(p))-f(p, \wp(p))]\right|\left|{ }^{C F} I[f(p, \hbar(p))-f(p, \wp(p))]+1\right| \\
& \leq\left(\frac{2(1-\varsigma)}{(2-\varsigma) M(\varsigma)}|f(p, \hbar(p))-f(p, \wp(p))|\right. \\
& \left.+\frac{2 \varsigma}{(2-\varsigma) M(\varsigma)} \int_{0}^{p}|f(p, \hbar(p))-f(p, \wp(p))| d p\right) \\
& \times\left(\frac{2(1-\varsigma)}{(2-\varsigma) M(\varsigma)}|f(p, \hbar(p))-f(p, \wp(p))|\right. \\
& \left.+\frac{2 \varsigma}{(2-\varsigma) M(\varsigma)} \int_{0}^{p}|f(p, \hbar(p))-f(p, \wp(p))| d p+1\right) \\
& \leq\left(\frac{2(1-\varsigma)}{(2-\varsigma) M(\varsigma)} \cdot \frac{(2-\varsigma) M(\varsigma)}{2} e^{-y}|\hbar(p)-\wp(p)|\right. \\
& \left.+\frac{2 \varsigma}{(2-\varsigma) M(\varsigma)} \cdot \frac{(2-\varsigma) M(\varsigma)}{2} \int_{0}^{p} e^{-y}|\hbar(p)-\wp(p)| d p\right) \\
& \times\left(\frac{2(1-\varsigma)}{(2-\varsigma) M(\varsigma)} \cdot \frac{(2-\varsigma) M(\varsigma)}{2} e^{-y}|\hbar(p)-\wp(p)|\right. \\
& \left.+\frac{2 \varsigma}{(2-\varsigma) M(\varsigma)} \cdot \frac{(2-\varsigma) M(\varsigma)}{2} \int_{0}^{p} e^{-y}|\hbar(p)-\wp(p)| d p+1\right) \\
& \leq\left(e^{-y} \sup _{p \in \mathfrak{J}}|\hbar(p)-\wp(p)|\right)\left(e^{-y} \sup _{p \in \mathfrak{J}}|\hbar(p)-\wp(p)|+1\right) \\
& =\left(e^{-y} d(\hbar, \wp)\right)\left(e^{-y} d(\hbar, \wp)+1\right) \\
& =\left(e^{-y} d(\hbar, \wp)\right)^{2}+\left(e^{-y} d(\hbar, \wp)\right) \\
& =e^{-y}\left[e^{-y}(d(\hbar, \wp))^{2}+d(\hbar, \wp)\right] \\
& \leq e^{-y}\left[(d(\hbar, \wp))^{2}+d(\hbar, \wp)\right] \text {. }
\end{aligned}
$$

Hence for $\hbar, \wp \in \mathcal{C}(\mathfrak{J}), p \in \mathfrak{J}$ with $J(\hbar(p), \wp(p)) \geq 0$, we have

$$
\begin{aligned}
& (d(\top \hbar(p), \top \wp(p)))^{2}+d(\top \hbar(p), \top \wp(p)) \\
& \leq e^{-y}\left[(d(\hbar, \wp))^{2}+d(\hbar, \wp)\right] .
\end{aligned}
$$


So

$$
\begin{aligned}
\operatorname{Ln} & {\left[(d(\top \hbar(p), \top \wp(p)))^{2}+d(\top \hbar(p), \top \wp(p))\right] } \\
& \leq \operatorname{Ln} e^{-y}+\operatorname{Ln}\left[(d(\hbar, \wp))^{2}+d(\hbar, \wp)\right],
\end{aligned}
$$

therefore

$$
\begin{aligned}
y+ & \operatorname{Ln}\left[(d(\top \hbar(p), \top \wp(p)))^{2}+d(\top \hbar(p), \top \wp(p))\right] \\
& \leq \operatorname{Ln}\left[(d(\hbar, \wp))^{2}+d(\hbar, \wp)\right] .
\end{aligned}
$$

Now, suppose $F:[0, \infty) \rightarrow R$ is defined by $F(u)=\operatorname{Ln}\left(u^{2}+u\right), u>0$, then it is straightforward to show that $F \in \mathfrak{F}$.

Set $\alpha: \mathcal{C}(\mathfrak{J}) \times \mathcal{C}(\mathfrak{J}) \rightarrow\{-\infty\} \cup[0, \infty)$ by

$$
\alpha(\hbar, \wp)= \begin{cases}1 & J(\hbar(p), \wp(p)) \geq 0, \text { for all } p \in \mathfrak{J} \\ -\infty & \text { else }\end{cases}
$$

then we have $y+\alpha(\hbar, \wp) F(d(\top \hbar, \top \wp)) \leq F(d(\hbar, \wp))$ for $\hbar, \wp \in M$ with $d(\top \hbar, \top \wp)>0$. Therefore we conclude that $T$ satisfies all conditions of definition of $\alpha$-type $F$-contraction. By $(\mathrm{H} 3)$,

$$
\begin{aligned}
\alpha(\hbar, \wp) \geq 1 & \Rightarrow \quad J(\hbar(p), \wp(p)) \geq 0 \quad \Rightarrow \quad J(\top(\hbar), \top(\wp)) \geq 0 \\
& \Rightarrow \alpha(\top(\hbar), \top(\wp)) \geq 1, \quad \hbar, \wp \in \mathcal{C}(\mathfrak{J}),
\end{aligned}
$$

which shows that $T$ is $\alpha$-admissible. From (H2) we have $\hbar_{0} \in \mathcal{C}(\mathfrak{J})$ such that $\alpha\left(\hbar_{0}, T \hbar_{0}\right) \geq 1$. According to (H4) and Theorem 2.3, we can obtain $\hbar^{*} \in \mathcal{C}(\mathfrak{J})$ where $\hbar^{*}=T \hbar^{*}$ which is a fixed point of $T$ and therefore a solution of the problem.

Now to use in the next definition let us define $\mathfrak{F}$ involved the functions $\psi:(0, \infty) \rightarrow R$ such that:

$\left(\mathfrak{F}_{1}\right) \quad 0<p<t$ implies $\psi(p) \leq \psi(t)$;

$\left(\mathfrak{F}_{2}\right) s_{n} \rightarrow 0$ if and only if $\psi\left(s_{n}\right) \rightarrow-\infty$, where $\left\{s_{n}\right\} \subset(0,+\infty)$.

Definition 2.5 ([20]) Let $\psi \in \mathfrak{F}, a \in[0,+\infty)$ and $d: M \times M \rightarrow[0,+\infty)$ with the following conditions:

$\left(d_{1}\right)(\hbar, \wp) \in M \times M, d(\hbar, \wp)=0 \Leftrightarrow \hbar=\wp ;$

$\left(d_{2}\right) d(\hbar, \wp)=d(\wp, \hbar)$, for $(\hbar, \wp) \in M \times M$;

$\left(d_{3}\right)$ If $(\hbar, \wp) \in M \times M,\left(u_{i}\right)_{i=1}^{N} \subset M$ such that $\left(u_{1}, u_{N}\right)=(\hbar, \wp), N \in \mathrm{N}, N \geq 2$ we have

$$
d(\hbar, \wp)>0 \quad \text { implies } \quad \psi(d(\hbar, \wp)) \leq \psi\left(\sum_{i=1}^{N-1} d\left(u_{i}, u_{i+1}\right)\right)+a
$$

Then $d$ is called an $\mathfrak{F}$-metric on $M$, and the pair $(M, d)$ is said to be a $\mathfrak{F}$-metric space. 
If we have the following condition for a sequence $\left\{\hbar_{n}\right\}$

$$
\lim _{n \rightarrow \infty} d\left(\hbar_{n}, \hbar\right)=0,
$$

then we say $\left\{\hbar_{n}\right\}$ is convergent to $\hbar$ with respect to $\mathfrak{F}$-metric $d$. Similar to the common definitions $\left\{\hbar_{n}\right\}$ is $\mathfrak{F}$-Cauchy in $(M, d)$ if

$$
\lim _{n, m \rightarrow+\infty} d\left(\hbar_{n}, \hbar_{m}\right)=0
$$

Similarly the $\mathfrak{F}$-completeness of $(M, d)$ can be defined.

Essential are the next definition and also the next fixed point theorem: let $\Gamma$ be the set of $\ell:[0, \infty) \rightarrow[0, \infty)$ such that

$\left(\ell_{1}\right) \quad \ell$ is nondecreasing;

$\left(\ell_{2}\right) \sum_{n=1}^{\infty} \ell^{n}(p)<\infty$ for $p \in R^{+}$, where $\ell^{n}$ is the $n$th iterate of $\ell$.

Definition 2.6 ([31]) Suppose that $\psi: M \rightarrow M$ and $\alpha: M \times M \rightarrow[0, \infty)$, if for $p \in M$

$$
\alpha(p, \psi p) \geq 1 \quad \Rightarrow \quad \alpha\left(\psi p, \psi^{2} p\right) \geq 1 .
$$

Then $\psi$ is said to be an $\alpha$-orbital admissible.

Theorem 2.7 ([13]) Suppose $(M, d)$ be a $\mathfrak{F}$-complete metric space and $\psi: M \rightarrow M$ such that

$$
\alpha(\hbar, \wp) d(\psi \hbar, \psi \wp) \leq \ell(d(\hbar, \wp))
$$

for $\hbar, \wp \in M$, where $\ell \in \Gamma$. Also assume

(n1) $\psi$ is $\alpha$-orbital admissible;

(n2) there exists $\hbar_{0} \in M$ with $\alpha\left(\hbar_{0}, \psi \hbar_{0}\right) \geq 1$;

(n3) $\psi \in \mathfrak{F}$ verifying $\left(d_{3}\right)$ is continuous and for a continuous function $\ell$ we have $\psi(u)>$ $\psi(\ell(u))+a, 0<u<\infty$, such that a is the same appeared in $\left(d_{3}\right)$.

Then there exists a fixed point for $f$.

Now let us define the $\mathfrak{F}$-metric $d: M \times M \rightarrow[0, \infty)$ given by

$$
d(\hbar, \wp)= \begin{cases}e^{|\hbar-\wp|} & \text { if } \hbar \neq \wp, \\ 0 & \text { if } \hbar=\wp,\end{cases}
$$

where $M=\mathcal{C}(\mathfrak{J}, \mathrm{N})$ and continues function $\psi$ on $(0, \infty)$ by $\psi(p)=-\frac{1}{p}$ for $p>0$. Since $-\frac{1}{u}>$ $\frac{1}{\ell(u)}>1$, it is obvious that $\psi(u)>\psi(\ell(u))+a, u>0$, in the sense that $\ell$ has the following conditions:

$$
\begin{aligned}
\ell(u) & <\frac{u}{u+1}, \\
e^{\ell(p)} & \leq \ell\left(e^{p}\right), \quad p \in\{0,1,2,3, \ldots\} .
\end{aligned}
$$

Now we are ready to present the following theorem. 
Theorem 2.8 Assume

(n1) there exists $\mathrm{j}: R^{2} \rightarrow R$ with

$$
|f(p, \hbar(p))-f(p, \wp(p))| \leq \frac{(2-\varsigma) M(\kappa)}{2} \ell(|\hbar(p)-\wp(p)|)
$$

where $s \in \mathfrak{J}$ and $\hbar, \wp \in R$ such that $J(\hbar, \wp) \geq 0$;

(n2) there exists $\hbar_{1} \in \mathcal{C}(\mathfrak{J})$ with $J\left(\hbar_{1}(p), \top \hbar_{1}(p)\right) \geq 0$ where $p \in \mathfrak{J}$ and $\top: \mathcal{C}(\mathfrak{J}) \rightarrow \mathcal{C}(\mathfrak{J})$ is defined by

$$
(\top \hbar)(p)=\hbar_{0}+{ }_{0}^{C F} I^{\varsigma} f(p, \hbar(p))
$$

(n3) for $p \in \mathfrak{J}$ and $\hbar \in \mathcal{C}(\mathfrak{J}), J(\hbar(p), \top \hbar(p)) \geq 0$ implies $J\left(\top \hbar(p), \top^{2} \hbar(p)\right) \geq 0$.

Then there exists at least one fixed point for $\top$ which is the solution of (6).

Proof We have

$$
\begin{aligned}
|\top \hbar(p)-\top \wp(p)|= & \left|{ }_{0}{ }_{0} I[f(p, \hbar(p))-f(p, \wp(p))]\right| \\
\leq & \left|\frac{2(1-\varsigma)}{(2-\varsigma) M(\varsigma)} \cdot \frac{(2-\varsigma) M(\varsigma)}{2} \ell\right| \hbar(p)-\wp(p) \mid \\
& +\frac{2 \varsigma}{(2-\varsigma) M(\varsigma)} \cdot \frac{(2-\varsigma) M(\varsigma)}{2} \int_{0}^{p} \ell|\hbar(p)-x(p)| d p \mid \\
\leq & (1-\varsigma+\varsigma)\left(\sup _{p \in \mathfrak{J}}|\hbar(p)-\wp(p)|\right. \\
\leq & \ell(|\hbar(p)-\wp(p)|) .
\end{aligned}
$$

Hence for $\hbar, \wp \in \mathcal{C}(\mathfrak{J}), p \in \mathfrak{J}$ with $J(\hbar(p), \wp(p)) \geq 0$, we have

$$
d(\top \hbar, \top \wp)=e^{|\top \hbar(p)-\top \wp(p)|} \leq e^{\ell(|\hbar(p)-\wp(p)|)} \leq \ell\left(e^{|\hbar(p)-\wp(p)|}\right)=\ell(d(\hbar, \wp)) .
$$

Put $\alpha: \mathcal{C}(\mathfrak{J}) \times \mathcal{C}(\mathfrak{J}) \rightarrow[0, \infty)$ by

$$
\alpha(\hbar, \wp)= \begin{cases}1 & J(\hbar(p), \wp(p)) \geq 0, p \in \mathfrak{J} \\ 0 & \text { else. }\end{cases}
$$

Therefore $\alpha(\hbar, \wp) d(\top \hbar, \top \wp)) \leq d(\top \hbar, \top \wp)) \leq \ell(d(\hbar, \wp)), \hbar, \wp \in M$ with $d(\top \hbar, \top \wp)>0$.

From (n3),

$$
\begin{aligned}
\alpha(\hbar, \top \hbar) \geq 1 \quad & \Rightarrow \quad(\hbar(p), \top \hbar(p)) \geq 0 \quad \Rightarrow \quad J\left(\top(\hbar), \top^{2}(\hbar)\right) \geq 0 \\
& \Rightarrow \quad \alpha\left(\top(\hbar), \top^{2}(\hbar)\right) \geq 1
\end{aligned}
$$

for $\hbar \in \mathcal{C}(\mathfrak{J})$. Therefore, we conclude that $\top$ is orbital $\alpha$-admissible. From (n2), we can choose $\hbar_{1} \in \mathcal{C}(\mathfrak{J})$ such that $\alpha\left(\hbar_{1}, \top \hbar_{1}\right) \geq 1$. Also from (n3) and Theorem 2.7, we get $\wp^{*} \in$ $\mathcal{C}(\mathfrak{J})$ with $\hbar^{*}=\top \hbar^{*}$. Hence, we obtain $\hbar^{*}$ as a solution of the problem and this completes the proof. 
Now, for a positive integer $n$ we denote by $h^{n}$ the $n$th iterate of $h$, so that $y=h^{0} y$ and $h^{n+1} y=h\left(h^{n} y\right)$ for $y \in \mathrm{X}$ and $n \in \mathrm{N}$. The triplet $(\mathrm{X}, d, h)$ represent a metric space $(\mathrm{X}, d)$ with a self-mapping $h$ on it. We shall use $(\mathrm{X} *, d, h)$ to indicate the corresponding metric space is complete. Also on $(\mathrm{X}, d, h)$ an orbit of $y_{0} \in \mathrm{X}$ is the set

$$
O\left(y_{0}\right)=\left\{h^{n} y_{0}: n=0,1,2, \ldots\right\}
$$

and $\rho\left(y_{0}\right)$ denote to the diameter of the set $O\left(y_{0}\right)$. Note that for any subset $B$ of $\mathrm{X}, \rho(B)=$ $\sup \{d(u, y): u, y \in B\}$ is the diameter of $B$. We shall use the triplet $\left(\mathrm{X}_{0_{*}}, d, h\right)$ if for some $y \in \mathrm{X}$, every Cauchy sequence from $O(y)$ converges in X. In this case, the corresponding space is called orbitally complete.

Corollary 2.9 For $(\mathrm{X}, d *, h)$ with $p: \mathrm{X} \rightarrow \mathrm{N}$, we suppose there exists $\tau>0$ such that for $v, w \in \mathrm{X}$

$$
d\left(h^{p(y)} y, h^{p(y)} w\right) \leq e^{-\tau} d(y, w)
$$

Assume there exists $y_{0} \in \mathrm{X}$ such that $0<\rho<\infty$. Moreover, $(\mathrm{X}, d)$ is h-orbitally complete. Then $h$ has a unique fixed point.

Theorem 2.10 On account of (6), we assume that

$$
|h(p, \hbar(p))-h(p, \wp(p))| \leq \frac{(2-\varsigma) M(\varsigma)}{2} e^{-y} \mid \sqrt{|\hbar(p)|}-\sqrt{|\wp(p)| \mid}
$$

and

$$
|h(p, \hbar(p))|+|h(p, \wp(p))| \leq \frac{(2-\varsigma) M(\varsigma)}{2} e^{-y} \mid \sqrt{|\hbar(p)|}+\sqrt{|\wp(p)| \mid},
$$

for $p \in \mathfrak{J}$, then the problem (6) possesses a unique solution.

Proof We consider $d: M \times M \rightarrow[0, \infty)$ given by $d(\hbar, \wp)=\sup _{p \in \mathfrak{J}}|\hbar(p)-\wp(p)|$ and, applying the Caputo-Fabrizio integral to both sides of (6), we get

$$
(\top \hbar)(p)=\hbar_{0}+{ }_{0}^{C F} I^{5} h(p, \hbar(p)) .
$$

We demonstrate that (6) has a unique solution. We get

$$
\begin{aligned}
|T \hbar(p)-T \wp(p)|= & \left|{ }^{C F} I[h(p, \hbar(p))-h(p, \wp(p))]\right| \\
\leq & \frac{2(1-\varsigma)}{(2-\varsigma) M(\varsigma)}|h(p, \hbar(p))-h(p, \wp(p))| \\
& +\frac{2 \varsigma}{(2-\varsigma) M(\varsigma)} \int_{0}^{p}|h(p, \hbar(p))-h(p, \wp(p))| d p \\
\leq & \frac{2(1-\varsigma)}{(2-\varsigma) M(\varsigma)} \cdot \frac{(2-\varsigma) M(\varsigma)}{2} e^{-y} \mid \sqrt{|\hbar(p)|}-\sqrt{|\wp(p)| \mid} \\
& +\frac{2 \varsigma}{(2-\varsigma) M(\varsigma)} \cdot \frac{(2-\varsigma) M(\varsigma)}{2} e^{-y} \int_{0}^{p} \mid \sqrt{|\hbar(p)|}-\sqrt{|\wp(p)|} d p
\end{aligned}
$$




$$
\leq e^{-y} \sup \mid \sqrt{|\hbar(p)|}-\sqrt{|\wp(p)| \mid},
$$

also

$$
\begin{aligned}
|\mathrm{T} \hbar(p)|+\left|\top_{\wp}(p)\right|= & \left|{ }^{C F} I[h(p, \hbar(p))]\right|+\left|{ }^{C F} I[h(p, \wp(p))]\right| \\
\leq & { }^{C F} I[|h(p, \hbar(p))|+|h(p, \wp(p))|] \\
\leq & \frac{2(1-\varsigma)}{(2-\varsigma) M(\varsigma)} \cdot \frac{(2-\varsigma) M(\varsigma)}{2} e^{-y} \mid \sqrt{|\hbar(p)|}+\sqrt{|\wp(p)| \mid} \\
& +\frac{2 \varsigma}{(2-\varsigma) M(\varsigma)} \cdot \frac{(2-\varsigma) M(\varsigma)}{2} e^{-y} \int_{0}^{p} \mid \sqrt{|\hbar(p)|}+\sqrt{|\wp(p)|} d p \\
\leq & e^{-y} \sup |\sqrt{|\hbar(p)|}+\sqrt{|\wp(p)|} \leq \sup | \sqrt{|\hbar(p)|}+\sqrt{|\wp(p)|} .
\end{aligned}
$$

On the other hand

$$
\sup (|\top \hbar(p)|+|\top \wp(p)|) \leq \sup \mid \sqrt{|\hbar(p)|}+\sqrt{|\wp(p)|},
$$

also we have

$$
\begin{aligned}
d\left(T^{2} \hbar, T^{2} \wp\right) & =\sup \left(\left|T^{2} \hbar(p)-T^{2} \wp(p)\right|\right) \\
& =\sup (|T \hbar(p)-T \wp(p)|) \times \sup (|T \hbar(p)+T \wp(p)|) \\
& \leq \sup (|T \hbar(p)-T \wp(p)|) \times \sup (|T \hbar(p)|+|T \wp(p)|) \\
& \leq e^{-y} \sup \sqrt{|\hbar(p)|}-\sqrt{|\wp(p)|} \times \sup \sqrt{|\hbar(p)|}+\sqrt{|\wp(p)|} \\
& =e^{-y} \sup || \hbar(p)|-| \wp(p)|| \\
& \leq e^{-y} \sup |\hbar(p)-\wp(p)| \\
& =e^{-y} d(\hbar, \wp) .
\end{aligned}
$$

Hence condition (10) holds with $p: \mathrm{X} \rightarrow \mathrm{N}$ such that $p(\hbar)=2, \hbar \in \mathrm{X}$. Accordingly all axioms of Corollary 2.9 are verified and consequently $\top$ possesses a unique fixed point. So (6) possesses a unique solution.

\section{Acknowledgements}

Not applicable.

Funding

Not applicable.

Availability of data and materials

Not applicable.

\section{Competing interests}

The authors declare that they have no competing interests.

Authors' contributions

All authors read and approved the final manuscript.

\section{Author details}

'Department of Mathematics, Faculty of Basic Sciences, University of Bonab, Bonab, Iran. ${ }^{2}$ Department of Applied Mathematics, Faculty of Mathematical Sciences, University of Tabriz, Tabriz, Iran. 


\section{Publisher's Note}

Springer Nature remains neutral with regard to jurisdictional claims in published maps and institutional affiliations.

Received: 15 April 2021 Accepted: 23 June 2021 Published online: 03 July 2021

\section{References}

1. Abdeljawad, T., Agarwal, R., Karapinar, E., Kumari, S.: Solutions of the nonlinear integral equation and fractional differential equation using the technique of a fixed point with a numerical experiment in extended b-metric space. Symmetry 11(5), 686 (2019)

2. Abdeljawad, T., Baleanu, D.: Integration by parts and its applications of a new nonlocal fractional derivative with Mittag-Leffler nonsingular kernel. J. Nonlinear Sci. Appl. 10, 1098-1107 (2017)

3. Adiguzel, R.S., Aksoy, U., Karapinar, E., Irhan, l.: On the solution of a boundary value problem associated with a fractional differential equation. Math. Methods Appl. Sci. (2020). https://doi.org/10.1002/mma.6652

4. Afshari, H., Alsulami, H.H., Karapinar, E.: On the extended multivalued Geraghty type contractions. J. Nonlinear Sci. Appl. 9, 4695-4706 (2016). https://doi.org/10.22436/jnsa.009.06.108

5. Afshari, H., Aydi, H., Karapinar, E.: Existence of fixed points of set-valued mappings in b-metric spaces. East Asian Math. J. 32(3), 319-332 (2016)

6. Afshari, H., Kalantari, S., Baleanu, D.: Solution of fractional differential equations via $\alpha-\psi$-Geraghty type mappings. Adv. Differ. Equ. 2018, 347 (2018)

7. Afshari, H., Kalantari, S., Karapinar, E.: Solution of fractional differential equations via coupled fixed point. Electron. J. Differ. Equ. 286, 1 (2015)

8. Aksoy, U., Karapinar, E., Erhan, l.: Fixed points of generalized alpha-admissible contractions on b-metric spaces with an application to boundary value problems. J. Nonlinear Convex Anal. 17(6), 1095-1108 (2016)

9. Algahtani, B., Aydi, H., Karapinar, E., Rakocevic, E.: A solution for Volterra fractional integral equations by hybrid contractions. Mathematics 7(8), 694 (2019)

10. Ali Shah, N., Khan, I.: Heat transfer analysis in a second grade fluid over and oscillating vertical plate using fractional Caputo-Fabrizio derivatives. Eur. Phys. J. C 76, 362 (2016). https://doi.org/10.1140/epjc/s10052-016-4209-3

11. Alqahtani, B., Fulga, A., Jarad, F., Karapinar, E.: Nonlinear F-contractions on b-metric spaces and differential equations in the frame of fractional derivatives with Mittag-Leffler kernel. Chaos Solitons Fractals 128, 349-354 (2019). https://doi.org/10.1016/j.chaos.2019.08.002

12. Arshad, M., Ameer, E., Karapinar, E.: Generalized contractions with triangular a-orbital admissible mapping on Branciari metric spaces. J. Inequal. Appl. 2016, 63 (2016)

13. Aydi, H., Karapinar, E., Mitrovi, Z.D., Rashid, T.: A remark on "Existence and uniqueness for a neutral differential problem with unbounded delay via fixed point results F-metric space". Rev. R. Acad. Cienc. Exactas Fís. Nat., Ser. A Mat. 113, 3197-3206 (2019). https://doi.org/10.1007/s13398-019-00690-9

14. Aydi, H., Karapinar, E., Yazidi, H.: Modified F-contractions via alpha-admissible mappings and application to integral equations. Filomat 31(5), 1141-1148 (2017)

15. Aydi, H., Karapinar, E., Zhang, D.: Common fixed points in the context of Brianciari metric spaces. Results Math. 71 73-91 (2017)

16. Caputo, M., Fabrizzio, M.: A new definition of fractional derivative without singular kernel. Prog. Fract. Differ. Appl. 1(2), 73-85 (2015)

17. Gopal, D., Abbas, M., Kumar, D.P., Vetro, C.: Fixed points of $\alpha$-type F-contractive mappings with an application to nonlinear fractional differential equation. Acta Math. Sci. 36(3), 957-970 (2016)

18. Goufo, E.F., Doungmo, P., Morgan, K., Mwambakana, J.N.: Duplication in a model of rock fracture with fractional derivative without singular kernel. Open Math. 13, 839-846 (2015)

19. Goufoa, D., Kumar, E.F., Mugisha, S.B.: Similarities in a fifth-order evolution equation with and with no singular kernel. Chaos Solitons Fractals 130, 109467 (2020)

20. Jeli, M., Samet, B.: On a new generalization of metric spaces. Fixed Point Theory Appl. 2018, 128 (2018)

21. Karapinar, E., Abdeljawad, T., Jarad, F.: Wardowski type contractions with applications on Caputo type nonlinear fractional differential equations. Adv. Differ. Equ. 2019, 421 (2019)

22. Karapinar, E., Fulga, A., Rashid, M., Shahid, L., Aydi, H.: Large contractions on quasi-metric spaces with an application to nonlinear fractional differential equations. Mathematics 7(5), 444 (2019)

23. Karapinar, E., Samet, B.: Generalized $\alpha$ - $\psi$-contractive type mappings and related fixed point theorems with applications. Abstr. Appl. Anal. 2012, Article ID 793486 (2012)

24. Kilbas, A., Srivastava, H.M., Trujillo, J.J.: Theory and Application of Fractional Differential Equations. North Holland Math Stud., vol. 204 (2006)

25. Losada, J., Nieto, J..: Properties of a new fractional derivative without singular kernel. Prog. Fract. Differ. Appl. 1(2), 87-92 (2015)

26. Marasi, H.R., Afshari, H., Daneshbastam, M., Zhai, C.B.: Fixed points of mixed monotone operators for existence and uniqueness of nonlinear fractional differential equations. J. Contemp. Math. Anal. 52, 8-13 (2017)

27. Marasi, H.R., Afshari, H., Zhai, C.B.: Some existence and uniqueness results for nonlinear fractional partial differential equations. Rocky Mt. J. Math. 47, 571-585 (2017). https://doi.org/10.1216/RMJ-2017-47-2-1

28. Marasi, H.R., Aydi, H.: Existence and uniqueness results for two-term nonlinear fractional differential equations via a fixed point technique. J. Math. 2021, Article ID 6670176 (2021). https://doi.org/10.1155/2021/6670176

29. Marasi, H.R., Karimi, S.: Convergence of the variational iteration method for solving fractional Klein-Gordon equation. J. Math. Comput. Sci. 4(2), 257-266 (2014)

30. Marasi, H.R., Nikbakht, M.: The Adomian decomposition method for boundary eigenvalue problems. Aust. J. Basic Appl. Sci. 5(12), 2106-2111 (2011)

31. Popescu, O.: Some new fixed point theorems for $\alpha$-Geraghty-contraction type maps in metric spaces. Fixed Point Theory Appl. 2014, 190 (2014)

32. Rezapour, S., Shabibi, M.: A singular fractional differential equation with Riemann-Liouville integral boundary condition. J. Adv. Math. Stud. 8(1), 80-88 (2015) 
33. Samet, B., Vetro, C., Vetro, P.: Fixed point theorems for $\alpha$ - $\psi$-contractive type mappings. Nonlinear Anal. 75 , 2154-2165 (2012)

34. Shatanawi, W., Karapinar, E., Aydi, H., Fulga, A.: Wardowski type contractions with applications on Caputo type nonlinear fractional differential equations. Sci. Bull. “Politeh.” Univ. Buchar., Ser. A, Appl. Math. Phys. 82(2), 157-170 (2020)

35. Wardowski, D.: Fixed point theory of a new type of contractive mappings in complete metric spaces. Fixed Point Theory Appl. 2012, 94 (2012)

Submit your manuscript to a SpringerOpen ${ }^{\circ}$ journal and benefit from:

- Convenient online submission

- Rigorous peer review

- Open access: articles freely available online

- High visibility within the field

- Retaining the copyright to your article

Submit your next manuscript at $\boldsymbol{\Delta}$ springeropen.com 